\title{
Gastric volvulus or just a large stomach? a case report with literature review of gastric volvulus and gastrointestinal pathology in acromegaly
}

\author{
Jasmine J. Mui*, Amit Sarkar, Mark Magdy
}

Department of General Surgery, The Sutherland Hospital, Caringbah, New South Wales, Australia

Received: 23 December 2019

Revised: 06 February 2020

Accepted: 07 February 2020

\author{
*Correspondence: \\ Dr. Jasmine J. Mui, \\ E-mail: jasmine.jm.mui@gmail.com
}

Copyright: () the author(s), publisher and licensee Medip Academy. This is an open-access article distributed under the terms of the Creative Commons Attribution Non-Commercial License, which permits unrestricted non-commercial use, distribution, and reproduction in any medium, provided the original work is properly cited.

\begin{abstract}
Gastric volvulus is a surgical emergency that requires prompt recognition and management. The acromegalic patient has a number of pathophysiological factors that predispose to gastric volvulus and slow gastrointestinal (GI) transit. Authors aimed to present a case of hiatus hernia and gastric volvulus in a patient with acromegaly and review the current literature on GI anomalies in this population. A 70 -year-old female presented to our institute with epigastric pain and coffee-ground vomiting on the background of acromegaly secondary to pituitary adenoma (resected in 1997). She was found to have a gastric volvulus and hiatus hernia which was repaired laparoscopically. She was discharged home but re-presented six days later with abdominal distension and vomiting. Computed tomography (CT) scan of abdomen showed recurrent gastric volvulus with involvement of the transverse colon. She underwent a laparotomy but no evidence of gastric or colonic volvulus was seen intra-operatively. The CT findings were attributed to a large stomach and coiled redundant transverse colon which could be misinterpreted as volvulus on imaging. Gastroparesis and slow bowel transit were the likely aetiology of this second presentation. This is consistent with literature reporting slow bowel transit and dolichocolon in acromegalic patients. Gastric volvulus is a rare finding associated with acromegaly. Structural anomalies in the anatomy of the acromegalic patient can make CT diagnosis challenging. This case demonstrates the need for caution when interpreting imaging in this cohort, as well as the need for further research on GI pathology associated with acromegaly.
\end{abstract}

Keywords: Acromegaly, Gastric volvulus, Hiatus hernia

\section{INTRODUCTION}

Gastric volvulus is a surgical emergency that requires prompt recognition and management to prevent gastric ischemia, necrosis and abdominal sepsis. Patients with acromegaly represent a distinct cohort with anatomical and physiological anomalies that predispose to gastric volvulus, slow gastrointestinal (GI) transit and hiatus hernia. ${ }^{1-4}$ Understanding the potential mechanisms behind such anomalies will enable better patient assessment, management and long-term care. Hence, authors present the case of a woman with acromegaly admitted with gastric volvulus secondary to hiatus hernia, who then represented with gastric outlet obstruction.

\section{CASE REPORT}

A 70-year-old female presented to institute with sudden onset epigastric pain and coffee ground vomitus. She has a medical history of acromegaly with pituitary adenoma resection in 1997, constipation, ischemic heart disease with 2 STEMIs and CABG, hypertension, hypercholesterolemia and osteoarthritis. The patient takes monthly octreotide injections, aspirin, clopidogrel, candesartan and rosuvastatin. She has a normal growth 
hormone $(\mathrm{GH})$ and insulin-like growth factor (IGF-1) level. On examination, the patient was hemodynamically stable with lactate of $2.4, \mathrm{Hb} 122$, urea 4.4 and WCC 9.28. She had epigastric tenderness and bowel sounds above the diaphragm.

Computed tomography (CT) of chest/abdomen/pelvis showed a large hiatus hernia (grade III) with gastric volvulus (Figure 1). A nasogastric (NG) tube was unable to be inserted past the area of volvulus despite attempts via gastroscopy. The patient proceeded to have a repeat gastroscopy and laparoscopy with hiatus hernia repair. Intraoperative findings included mesenteroaxial gastric volvulus with the entire stomach in the thoracic cavity. There was contusion to the mid body of the stomach but no ischemia. The hiatus hernia was repaired with BioA mesh and an NG was successfully inserted via gastroscopy.

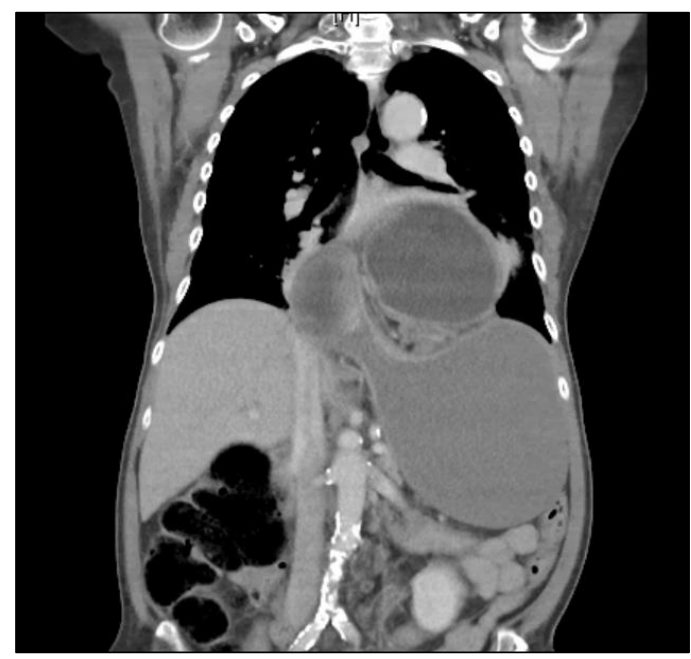

Figure 1: Coronal slice of CT chest/abdomen from first admission demonstrating hiatus hernia.

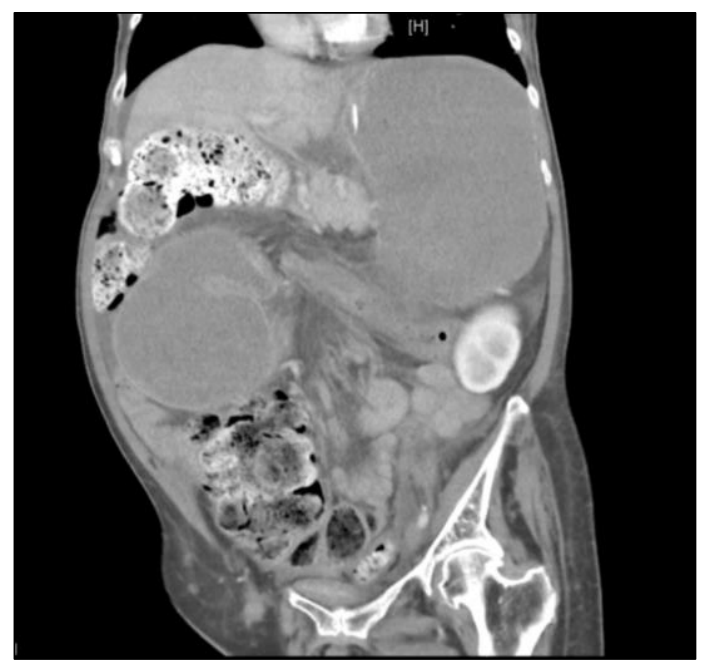

Figure 2. Coronal slice of CT Abdomen from second admission demonstrating apparent volvulus involving stomach and transverse colon.

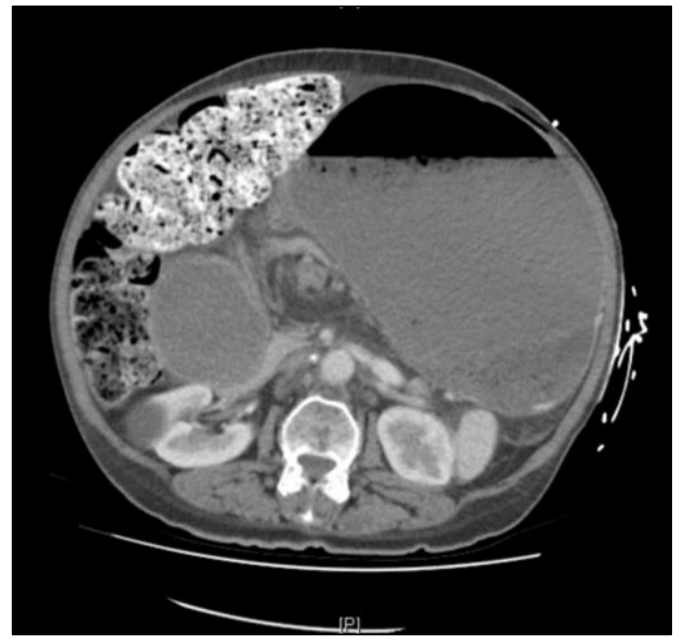

Figure 3: Axial slice of CT Abdomen from second admission demonstrating mesenteric swirl.

The patient had an uneventful post-operative admission and was discharged home day 5 post op on a soft puree diet. She re-presented six days later with vomiting and abdominal distension. A CT abdomen showed gastric obstruction secondary to mesenteric volvulus involving both stomach and transverse colon (Figures 2 and 3). There was no resolution of volvulus on repeat CT scan post NG insertion despite 3L of fluid drained from the NG. The patient admitted that she had not followed a strict puree diet post-discharge.

She was taken back to theatre for gastroscopy and laparoscopy which showed intact mesh repair with no evidence of gastric or transverse colon volvulus. There was gross dilation of the stomach suggestive of gastroparesis, and a redundant transverse colon loaded with faeces suggestive of slow colonic transit. On gastroscopy, significant amounts of liquid and semisolid content were visualized but unable to be aspirated and decompressed. The procedure was converted to a laparotomy with three-point gastric plication to the anterior abdominal wall. A 24 Fr gastrostomy tube was inserted and there was successful decompression with drainage of stomach contents. Postoperatively the patient was commenced on prokinetics to improve gastric motility. Bowels were opening day 11 post op and she was discharged home day 12 post op on a puree diet.

\section{DISCUSSION}

Acromegaly is a condition characterized by excess GH secretion resulting high circulating IGF-1, often secondary to a pituitary somatotroph adenoma. ${ }^{1}$ Acromegalics have distinctly large facial features, large hands and feet, and organomegaly. They are prone to the development of hypertension, arthropathy, diabetes mellitus and cardiovascular and respiratory disease. ${ }^{1-4}$ While bowel cancer is well documented in acromegalics, there is limited literature on the effect of acromegaly on the physiology and anatomy of the GI tract. ${ }^{3,4}$ This is the 
only case report of an acromegalic patient who has presented with a hiatus hernia and gastric volvulus.

Hiatus hernia is reported to be uncommon in acromegalic patients. A Turkish small centre study found that in a cohort of 39 patients with acromegaly $7.6 \%$ had a hiatus hernia, which is lower than the prevalence in the general population. ${ }^{5}$ However, the acromegalic patient does have risk factors for development of hiatus hernia such a respiratory muscle weakness and increased intraabdominal pressure. ${ }^{6}$ This demonstrates the need for further research in this area with larger cohort studies.

Patients with acromegaly have a prolonged small and large bowel transit time. ${ }^{7,8}$ A number of randomized control trials have demonstrated an increase in transit time from mouth to caecum and caecum to anus when comparing controls to acromegalic patients with normal GH and IGF-1 levels. ${ }^{7,8}$ Transit time was further increased by $71 \%$ when the acromegalic was taking octreotide. $^{7}$ Although the exact mechanism is not well understood, the underlying mechanism may be related to alterations in autonomic function, as colonic lengthening has been shown to decrease bowel motility due to neuronal inhibition of colonic migrating motor complexes. ${ }^{8-10}$ Hence autonomic dysregulation and small and large bowel lengthening from the effect of IGF-1 predisposes acromegalics to the development of slow bowel transit, which is exacerbated by octreotide use. While there is no data on gastroparesis in acromegalics, autonomic dysregulation is a hallmark of gastroparesis and it is likely that the autonomic dysfunction in the bowel is also present in the stomach. ${ }^{11}$ In patient, the intraoperative findings of a large, dilated stomach full of static contents suggests chronic gastroparesis. The severe faecal loading of the transverse colon is consistent with slow bowel transit.

Dolichocolon has been the proposed mechanism behind two case reports of sigmoid volvulus in the acromegalic patient and one case report of total colonic volvulus. ${ }^{12-13}$ Organomegaly secondary to the effects of IGF-1 results in a long and redundant sigmoid colon prone to volvulus around a narrow mesocolon. ${ }^{13}$

For patient, the mechanism of her gastric volvulus is potentially similar to that of a sigmoid volvulus as the enlarged stomach with narrow mesenteric attachment is prone to twisting on the mesenteric axis. Furthermore, gastromegaly and a large redundant transverse colon was the likely pathology behind the gastric mesenteric swirl sign and coiled transverse colon seen on CT scan during her second admission, rather than re-volvulus as there was no evidence of this intra-operatively. Therefore, patient had gastric outlet obstruction secondary to gastroparesis and slow bowel transit, not gastric revolvulus despite convincing CT signs. This indicates the need for caution in interpreting anomalies on imaging of the acromegalic patient.

\section{CONCLUSION}

This case report highlights an important variation in anatomy and physiology for patients with acromegaly, and how this can predispose to certain GI pathologies and misinterpretation of imaging. While acromegaly does appear to predispose to hiatus hernia, gastroparesis and gastric volvulus, further research is warranted to determine the underlying mechanism and enable adequate screening and prevention in this population.

\section{ACKNOWLEDGEMENTS}

The author would like to thank the subject of this case report for allowing us to publish their case to enrich the current literature in this field.

\section{Funding: No funding sources \\ Conflict of interest: None declared \\ Ethical approval: Not required}

\section{REFERENCES}

1. Renehan ABP, Painter J, O’Dwyer S, Haboubi N, Varma J, Ball S, et al. The prevalence and characteristics of colorectal neoplasia in acromegaly. J Clini Endocrinol Metabo. 2000;85:3417-24.

2. Melmed S. Acromegaly. In: Melmed S, ed. The Pituitary. $4^{\text {th }}$ ed: Academic Press; 2017:423-466.

3. Abreu A, Tovar AP, Castellanos R, Valenzuela A, Giraldo CM, Pinedo AC, et al. Challenges in the diagnosis and management of acromegaly: a focus on comorbidities. Pituitary. 2016;19(4):448-57.

4. Colao A, Auriemma RS, Pivonello R, Galdiero M, Lombardi G. Medical consequences of acromegaly: what are the effects of biochemical control?. Rev Endo Metab Dis. 2008;9(1):21-31.

5. Sisman P, Pekgoz M, Bayrakci I, Sisman M, Cander $\mathrm{S}$, Gul OO, et al. Evaluation of upper gastrointestinal system in acromegaly. Annale d'endocrinol. 2019;80(4):196-201.

6. George B, Vinay D, Moolechery J, Mathew V, Anantharaman R, Ayyar V, et al. Megaduodenum in a patient with acromegaly. Ind J Endocrinol Metab. 2012;16(2):324-5.

7. Veysey MJ, Thomas LA, Mallet AI, Jenkins PJ, Besser GM, Wass JA, et al. Prolonged large bowel transit increases serum deoxycholic acid: a risk factor for octreotide induced gallstones. Gut. 1999 May 1;44(5):675-81.

8. Resmini E, Parodi A, Savarino V, Greco A, Rebora A, Minuto F, et al. Evidence of prolonged orocecal transit time and small intestinal bacterial overgrowth in acromegalic patients. J Clin Endocrinol Metab. 2007;92(6):2119-24.

9. Southwell BR. Colon lengthening slows transit: is this the mechanism underlying redundant colon or slow transit constipation?. J Physiol. 2010;588(18):3343. 
10. Heredia DJ, Dickson EJ, Bayguinov PO, Hennig GW, Smith TK. Colonic elongation inhibits pellet propulsion and migrating motor complexes in the murine large bowel. J Physiol. 2010;588(15):291934.

11. Liu N, Abell T. Gastroparesis updates on pathogenesis and management. Gut Liver. 2017;11(5):579.

12. Hancerliogullari O, Senocak R, Kaymak S, Lapsekili E, Sinan H. An uncommon cause of acute abdomen in an acromegalic patient: colonic volvulus. Ann Ital Chir. 2018;89:572-6.
13. Chowdhury FH, Kawsar KA, Haque MR, Haque AF. Sigmoid Volvulus in Acromegalic Patient; Successful Surgical Management of Sigmoid Volvulus and Pituitary Macroadenoma: A Case Report. J Med. 2014;15(1):84-6.

Cite this article as: Mui JJ, Sarkar A, Magdy M. Gastric volvulus or just a large stomach? a case report with literature review of gastric volvulus and gastrointestinal pathology in acromegaly. Int Surg J 2020;7:941-4 